\title{
Aspectos sobre a biologia de Tonatia bidens (Spix) no estado do Rio de Janeiro, sudeste do Brasil (Mammalia, Chiroptera, Phyllostomidae)
}

\author{
Carlos E. L. Esbérard ${ }^{1,2} \&$ Helena G. Bergallo ${ }^{1}$ \\ ${ }^{1}$ Departamento de Ecologia, Universidade do Estado do Rio de Janeiro. Rua São Francisco Xavier 524, 20559-900 Rio de \\ Janeiro, Rio de Janeiro, Brasil. \\ 2 Fundação RIOZOO. Quinta da Boa Vista, 20940-040 Rio de Janeiro, Rio de Janeiro, Brasil. E-mail: cesberard@terra.com.br
}

\begin{abstract}
Biological aspects of Tonatia bidens (Spix) in Rio de Janeiro State, southeastern Brazil (Mammalia, Chiroptera, Phyllostomidae). The biology of Tonatia bidens (Spix, 1823) was studied at Rio de Janeiro state, southeastern Brazil. A total of 76 bats was captured using mist nets or using hand nets during sampling in diurnal roosts. Some observations of bats in captivity are also included. Males comprised $47 \%$ of the captures. Pregnant females were observed in November and May and lactant females in January, April and May. Subadult animals were recorded in December, February, May and July. The prey remains observed in feeding roosts demonstrates the insectivorous and carnivorous diet of this bats. The feeding behavior is similar to other carnivorous species of phyllostomid bats.
\end{abstract}

KEY WORDS. Daily activity, feeding behavior, occurrence, reproduction.

RESUMO. A biologia de Tonatia bidens (Spix, 1823) foi estudada no Estado do Rio de Janeiro, sudeste do Brasil. Um total de 76 morcegos foi capturado usando redes de neblina e redes manuais para insetos em refúgios diurnos. Algumas observações dos morcegos em cativeiro foram incluídas. Machos compreenderam 47\% das capturas. Fêmeas prenhas foram observadas em novembro e maio, e fêmeas lactantes em janeiro, abril e maio. Animais subadultos foram registrados em dezembro, fevereiro, maio e julho. Os restos de presas observadas em refúgios de alimentação demonstram a dieta insetívora e carnívora deste morcego. O comportamento alimentar é similar a de outras espécies carnívoras de morcegos Phyllostomidae.

PALAVRAS CHAVE. Atividade diária, comportamento alimentar, ocorrência, reprodução.

Tonatia bidens (Spix, 1823) é uma espécie de morcego que ocorre no leste e sudeste do Brasil, Paraguai e norte da Argentina (Williams et al. 1995). Registros dessa espécie no Brasil incluem os estados da Bahia, Mato Grosso (Williams et al. 1995), São Paulo (Martuscelli 1995, Vieira 1942, Williams et al. 1995), Espírito Santo (Ruschi 1953) e Rio de Janeiro (Peracchi \& Albuquerque 1986). Estes morcegos utilizam-se de ocos de árvores e cavernas para refúgio (PINTO-DA-ROCHA 1995, TutLE 1976, Villa-R. 1966), formando grupos pouco numerosos (Fenton \& Kunz 1977, Tutle 1976). A dieta conhecida de Tonatia bidens é composta principalmente de frutos e insetos (GARDNER 1977, Goodwin \& Greenhall 1961, Myers \& Wetzel 1983, Ruschi 1953), mas, relatos recentes descreveram a utilização de aves por este morcego (BARquez et al. 1999, MartusCelli 1995).

Morcegos do gênero Tonatia Gray, 1827 são pouco amostrados na natureza, sendo por vezes considerados raros (WILLIG, 1983), conseqüentemente, a biologia da espécie é pouco conhecida, baseada em dados fragmentados e pontuais (e.g. BARQUEZ et al. 1999, NoWAK 1995).
Aspectos sobre a biologia de T. bidens, como reprodução, atividade, parasitismo e alimentação são aqui relatados.

\section{MATERIAL E MÉTODOS}

As capturas dos morcegos foram realizadas com redes de neblina em trilhas ou clareiras existentes em florestas, junto a árvores em frutificação ou ainda sobre ou próximas a coleções de água. As redes permaneceram abertas entre os crepúsculos vespertino e matutino, sendo vistoriadas a intervalos médios de 20 minutos. Eventualmente, foram empregadas redes armadas nos acessos aos refúgios conhecidos de morcegos Phyllostomidae, Vespertilionidae e Molossidae, localizados em construções, ocos de árvores, grutas ou cavernas. Quando necessário usamos puçás entomológicos para captura no interior de abrigos. Entre abril de 1989 e dezembro de 2002, uma longa e representativa amostragem de morcegos (cerca de 700 noites, 57 diferentes localidades, mais de 18.000 capturas) foi realizada no Estado do Rio de Janeiro. Nesta amostragem foram 
analisadas tanto áreas degradadas, como vias públicas, quintais e parques urbanos, quanto florestas secundárias e áreas agrícolas.

Os animais capturados tiveram seus horários de captura anotados e comparados com o horário de pôr-do-sol do dia de captura. Os pesos dos morcegos foram obtidos com o uso de dinamômetro Pesola ${ }^{\circledR}$ (escala 1,0 g) ou balança de precisão Ouhaus $^{\circledR}$ (escala $0,1 \mathrm{~g}$ ) e o comprimento do antebraço medido com auxílio de paquímetro digital (escala $0,1 \mathrm{~mm}$ ). A condição reprodutiva das fêmeas foi analisada no momento da captura e cada exemplar capturado foi incluído em uma das seguintes categorias: fêmea inativa, fêmea com feto palpável, fêmea lactante, fêmea com mamilos intumescidos e não secretantes, fêmea simultaneamente grávida e lactante. Os machos foram separados em macho com testículos abdominais ou macho com testículos escrotados. Os animais foram considerados adultos ou jovens baseados na ossificação das epífises e na coloração do pelame (ANTHONY 1988).

A partir de 1997, ectoparasitas foram capturados manualmente ou com pinça de relojoeiro e armazenados em álcool a 70\% (WHITAKER 1988).

A maior parte dos exemplares foi marcada com o uso de coleiras plásticas providas de cilindros coloridos e soltos imediatamente após a captura (EsbérARd \& DAEMON 1999). Seis indivíduos foram sacrificados e depositados como material testemunho na coleção de referência da Fundação RIOZOO.

O comportamento alimentar de Tonatia bidens foi analisado por meio dos restos de presas encontrados nos poleiros de alimentação. Só foram considerados para análise os poleiros de alimentação que estavam em uso no período noturno.

As observações sobre o comportamento alimentar foram realizadas em animais mantidos em cativeiro. Esta espécie é mantida em cativeiro no Zoológico do Rio de Janeiro desde 1998. Os animais foram mantidos em gaiolas de $90 \times 60 \times 80$ $\mathrm{cm}$ de tela de arame de $1 / 2$ " e alimentados com camundongos jovens recém-abatidos e artrópodes secos (Cedé $\left.{ }^{\circledR}\right)$. Os animais foram mantidos em haréns ou grupos de fêmeas, com grupos não excedendo seis animais por gaiola. As gaiolas foram mantidas em sala com fotoperíodo natural, complementado no segundo semestre de cada ano até 14 horas de claro a cada dia através de lâmpadas fluorescentes. A temperatura foi mantida entre 26 e $31^{\circ} \mathrm{C}$ e a umidade de 70 a $85 \%$ (EsBÉRARD 2002, EsbÉRARd \& GOMES 2001).

Estação Ecológica Paraíso, $\left(22^{\circ} 29^{\prime} 22,2^{\prime \prime}\right.$ S e $042^{\circ} 54^{\prime}$ $\left.50,3^{\prime \prime} \mathrm{W}\right)$, Município de Guapimirim, centro do Estado do Rio de Janeiro, cerca de $80 \mathrm{~m}$ de altitude.

Material examinado: uma fêmea em 18/IV/1997; cinco machos e oito fêmeas em 16/V/1997; duas fêmeas em 20/VI/ 1997; uma fêmea em 05/IX/1997 e uma fêmea em 07/XI/1997.

Fazendas Reunidas São João, (22 33'01,98"S e $042^{\circ}$ 03'01,2”W), Município de Casimiro de Abreu, centro do Estado do Rio de Janeiro, cerca de $20 \mathrm{~m}$ de altitude.

Material examinado. BraziL, Rio de Janeiro: Município de
Guapimirim (Estação Ecológica Paraíso, 22²9'22,2"S e 04254' $50,3^{\prime \prime} \mathrm{W}$, cerca de $80 \mathrm{~m}$ de altitude), uma fêmea em 18/IV/1997, cinco machos e oito fêmeas em 16/V/1997, duas fêmeas em 20/ VI/1997, uma fêmea em 05/IX/1997 e uma fêmea em 07/XI/ 1997; Município de Casimiro de Abreu (Fazendas Reunidas São João, $22^{\circ} 33^{\prime} 01,98^{\prime \prime}$ S e $042^{\circ} 03^{\prime} 01,2^{\prime \prime} \mathrm{W}$, cerca de $20 \mathrm{~m}$ de altitude), um macho e duas fêmeas em 30/V/1997; dois machos e quatro fêmeas em 31/V/1997; um macho em 20/XI/1998; um macho em 22/I/1999; dois machos em 05/VIII/2000 e um macho e uma fêmea em 13/VII/2001; Município de Mangaratiba (Reserva Ecológica Rio das Pedras, $22^{\circ} 59^{\prime} 18,90^{\prime \prime} \mathrm{S}$ e $044^{\circ} 06^{\prime}$ 04,06"W), cerca de $30 \mathrm{~m}$ de altitude); duas fêmeas em 06/VI/ 1997; Município do Rio de Janeiro (Parque Nacional da Tijuca, $22^{\circ} 57^{\prime} 40,6^{\prime \prime} \mathrm{S}$ e $043^{\circ} 17^{\prime} 61,0^{\prime \prime} \mathrm{W}$, Maciço da Tijuca, no Açude da Solidão, $345 \mathrm{~m}$ de altitude), dois machos e uma fêmea em 27/ VI/1997, duas fêmeas em 18/VII/1997, um macho em 03/IV/ 1998 e um macho em 01/XII/1999; (Área de Proteção Ambiental

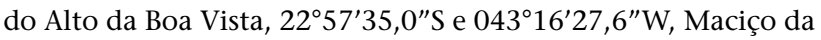
Tijuca, refugiados em minas d'água ou próximos a este refúgio, 365 m de altitude), um macho e uma fêmea em 29/I/2001 e seis fêmeas em 28/IX/2002; (Parque da Gávea, 2358'68,7"S e $043^{\circ} 14^{\prime} 54,3^{\prime \prime} \mathrm{W}$, Maciço da Tijuca, $98 \mathrm{~m}$ de altitude), um macho e uma fêmea em 21/I/1993; um macho em 01/XII/1993; (Reserva dos Trapicheiros, $22^{\circ} 56^{\prime} 11,3^{\prime \prime} \mathrm{S}$ e $043^{\circ} 14^{\prime} 04,1^{\prime \prime} \mathrm{W}$, Maciço da Tijuca, $76 \mathrm{~m}$ de altitude), dois machos em 16/II/1993; um macho em 08/IX/1993; (Jardim Botânico do Rio de Janeiro, $22^{\circ} 58^{\prime} 14,3^{\prime \prime}$ S e $043^{\circ} 13^{\prime} 43,0^{\prime \prime} \mathrm{W}$, Maciço da Tijuca, $30 \mathrm{~m}$ de altitude), um macho em 09/IX/1991, um macho em 21/IX/1994 e um macho e três fêmeas em 10/IV/1995; (Reserva Florestal do

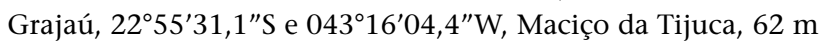
de altitude), um macho em 11/IV/1991; (Parque do Penhasco

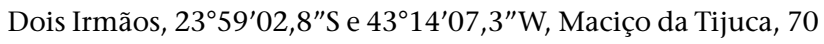
$\mathrm{m}$ de altitude), um macho em 01/XII/1999; (Parque Lage, $22^{\circ}$ 57'37,52"S e $043^{\circ} 12^{\prime} 41,83^{\prime \prime} \mathrm{W}$, Maciço da Tijuca, 4 m de altitude), dois machos e uma fêmea em 04/VII/1997 e um macho em 12/VIII/1998; (Reserva do Horto Florestal, 2257'33,60"S e $43^{\circ} 14^{\prime} 6,12^{\prime \prime} \mathrm{W}$, Maciço da Tijuca, a cerca de $35 \mathrm{~m}$ de altitude), um macho em 02/V/2000; Município de Angra dos Reis, (Ilha Grande, Vila Dois Rios, $23^{\circ} 10^{\prime} 32,46^{\prime \prime}$ e e $044^{\circ} 11^{\prime} 21,96^{\prime \prime} \mathrm{W}$, ao nível do mar), dois machos em 13/XII/1997 e dois machos em 11/ $\mathrm{XI} / 2002$; (Parque Estadual da Ilha Grande, Praia do Aventureiro, $23^{\circ} 10^{\prime} 48,26^{\prime \prime}$ S e $044^{\circ} 20^{\prime} 32,53^{\prime \prime} \mathrm{W}$, ao nível do mar), um macho em 26/I/1992; dois machos e três fêmeas em 29/I/1992; Município de Paraty (Área de Proteção Ambiental de Joatinga, Praia do sono ( $23^{\circ} 12^{\prime} 53,13^{\prime \prime}$ e e $044^{\circ} 39^{\prime} 46,58^{\prime \prime} \mathrm{W}$, ao nível do mar), um macho em 09/VI/1995.

\section{RESULTADOS}

\section{Ocorrência}

A espécie Tonatia bidens foi capturada em 15 das 57 localidades amostradas $(26,16 \%)$ até o momento no Estado do Rio de Janeiro (Fig. 1). Esteve ausente de todas as amostragens em 


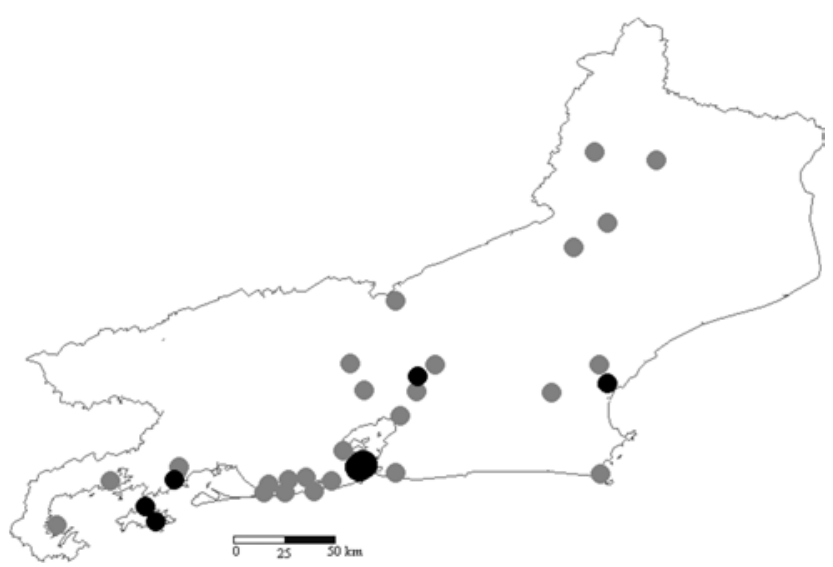

Figura 1. Localidades amostradas no Estado do Rio de Janeiro. Os pontos cinzas representam localidades onde Tonatia bidens não foi capturada, os pontos pretos onde a espécie foi capturada e o maior ponto negro representa o Maciço da Tijuca, Município do Rio de Janeiro, onde 29 localidades foram amostradas, sendo que em nove houve a confirmação da espécie.

áreas urbanas ( $\mathrm{N}=11$ localidades) e sua ocorrência pode ser descrita como mais freqüente em bordas de florestas, tendo também sido observada em vegetação característica de restinga, capoeiras e plantações de bananas, desde que limítrofes a matas.

Tonatia bidens correspondeu a menos de $0,4 \%$ de todas as capturas analisadas. Das 76 capturas analisadas, $48,5 \%$ ocorreram em redes armadas em clareiras, 13,2\% em redes armadas sobre ou próximas a coleções de água e as demais capturas ocorreram em trilhas, junto a refúgios ou nos poleiros de alimentação. A espécie foi mais freqüente em coletas realizadas em baixas altitudes (menos de $100 \mathrm{~m}$ ) e esteve ausente de inventários com mais de 1.000 capturas realizados em altitudes médias (cerca de 600 m, como Reserva Serra do Mar) e altas (1.100 m, como Reserva Biológica de Araras, Petrópolis).

\section{Biometria}

O comprimento do antebraço variou de $48,80 \mathrm{~mm}$ a 58,84 $\mathrm{mm}$, tendo as fêmeas em média 52,26 $\pm 1,05 \mathrm{~mm}$ (variando de 50,00 a $55,80 \mathrm{~mm}$ ) e os machos 52,99 $\pm 2,17 \mathrm{~mm}$ (variando de 48,80 a $58,84 \mathrm{~mm}$ ). Os machos foram significativamente maiores $(\mathrm{t}=-2,220, \mathrm{p}=0,034)$. $\mathrm{O}$ peso variou de 18 a $38 \mathrm{~g}$, com média de $28,29 \mathrm{~g} \pm 3,69 \mathrm{~g}$, para fêmeas e 27,93 $\pm 3,56 \mathrm{~g}$ para machos.

Reprodução. Dos 76 exemplares analisados, 34 eram machos $(44,7 \%)$. Exemplares com testículos aparentes compreenderam $38,2 \%$ (13 capturas) e foram observados nos meses de janeiro $(\mathrm{N}=2)$, março $(\mathrm{N}=2)$, abril $(\mathrm{N}=1)$, maio $(\mathrm{N}=3)$, junho $(\mathrm{N}=2)$, setembro $(\mathrm{N}=1)$, novembro $(\mathrm{N}=2)$ e dezembro $(\mathrm{N}=1)$.

Das fêmeas capturadas $(57,5 \%)$, confirmamos a prenhez pela presença de feto palpável, no mês de novembro $(\mathrm{N}=4)$. Fêmeas lactantes foram analisadas nos meses de janeiro $(\mathrm{N}=2)$, abril $(\mathrm{N}=2)$ e maio $(\mathrm{N}=1)$ e fêmeas pós-lactantes, com mami- los maiores que $2 \mathrm{~mm}$, em maio $(\mathrm{N}=3)$. Exemplares subadultos foram capturados em dezembro, fevereiro, maio e junho. Seis fêmeas capturadas, quatro das quais com fetos palpáveis, completaram a gestação em cativeiro, apresentando partos em 12/ XI/1999, 12/XII/2002, 28/XII/2002, 03/I/2003 e duas em 10/I/ 2003. O intervalo entre a captura e os partos variou de 75 a 104 dias, demonstrando ser a gestação superior a 3,5 meses. Apenas um dos neonatos foi mensurado e apresentou, na idade de sete dias, 9,0 g de peso e comprimento de antebraço de $32,65 \mathrm{~mm}$. Isso representa $32,4 \%$ do peso médio de fêmeas e $62,8 \%$ do comprimento médio de antebraço de fêmeas.

Tabela I. Espécies de Streblidae capturadas e identificadas parasitando Tonatia bidens no Estado do Rio de Janeiro. (EEEP) Estação Ecológica Estadual Paraíso, (PHL) Parque Henrique Lage, (PNT) Parque Nacional da Tijuca, (IGD) Ilha Grande; (M) macho, (F) fêmea, (L) larva, (X) presente em amostra composta por mais de um morcego.

\begin{tabular}{lcccc}
\hline \multicolumn{1}{c}{ Espécie de ectoparasita } & EEEP & PHL & PNT & IGD \\
\hline Strebla mirabilis (Waterhouse, 1879) & $1 \mathrm{~F}$ & & & \\
Strebla sp. & $1 \mathrm{M}$ & $1 \mathrm{M}$ & & \\
Trichobius complexo parasiticus & $1 \mathrm{M} 1 \mathrm{~F}$ & & $1 \mathrm{~L}$ & $\mathrm{X}$ \\
Trichobius dugesioides Wenzel, 1966 & $9 \mathrm{M} 7 \mathrm{~F}$ & & & \\
Trichobius sp. & $1 \mathrm{M}$ & & & \\
\hline
\end{tabular}

\section{Ectoparasitas}

Quatro espécies de ectoparasitas foram até o momento identificadas a partir de 12 espécimes, da Ordem Diptera (Streblidae) (Tab. I).

\section{Refúgios diurnos}

No Parque Estadual da Ilha Grande (Município de Angra dos Reis), T. bidens foi coletada junto a refúgio situado em oco de palmeira, a cerca de sete metros de altura, onde dois machos e três fêmeas (uma grávida e duas lactantes) foram coletadas. Esta espécie foi capturada na Reserva Florestal do Grajaú, Município do Rio de Janeiro, em gruta, coabitando com Carollia perspicillata (Linnaeus, 1758) e Desmodus rotundus (É. Geoffroy, 1810). Em gruta no interior do Parque Nacional da Tijuca (Município do Rio de Janeiro) esta espécie foi observada refugiada em três ocasiões, variando o número de um a até cinco animais. Na Área de Proteção Ambiental do Alto da Boa Vista, Município do Rio de Janeiro, foi capturada em mina d'água com cerca de $12 \mathrm{~m}$ de comprimento e $0,75 \mathrm{~m}$ de largura, coabitando com um exemplar de Tonatia silvicola (d'Orbigny, 1836). Em outra coleta no mesmo local foi observado grupo composto por sete exemplares, dos quais seis fêmeas foram capturadas, três das quais com fetos palpáveis. Neste local observamos alternância de espécies utilizando este refúgio. Após a remoção de três grupos de Desmodontinae (Desmodus rotundus e Diphylla ecaudata), T. bidens voltou a utilizar o local (Tab. II). 
Tabela II. Alternância de espécies refugiadas em mina d’água amostrada no município do Rio de Janeiro.

\begin{tabular}{cclc}
\hline Data & Intervalo (dias) & \multicolumn{1}{c}{ Espécies } & Quantidade \\
\hline $10 /$ III/2001 & 0 & Tonatia bidens & 2 \\
& & Tonatia silvicola & 1 \\
$28 / \mathrm{X} / 2001$ & 232 & Diphylla ecaudata & 3 \\
$10 / \mathrm{XI} / 2001$ & 13 & Desmodus rotundus & 10 \\
$22 / \mathrm{II} / 2002$ & 102 & Diphylla ecaudata & 3 \\
$22 / \mathrm{VI} / 2002$ & 128 & Vazio & \\
$10 / \mathrm{XI} / 2002$ & 140 & Tonatia bidens & 7 \\
$19 / \mathrm{VII} / 2003$ & 251 & Desmodus rotundus & 2 \\
& & Carollia perspicillata & 1 \\
\hline
\end{tabular}

\section{Poleiros de Alimentação}

Esta espécie foi capturada enquanto utilizava vários refúgios de alimentação. A presença de T. bidens pode ser constatada pela presença de restos das presas que consome, como insetos e pequenos vertebrados. Cinco tipos de poleiros de alimentação em uso foram encontrados: manilha $(\mathrm{N}=1)$, construção abandonada $(\mathrm{N}=1)$, construção em uso $(\mathrm{N}=2)$, oco de árvore $(\mathrm{N}=1)$, gruta $(\mathrm{N}=1)$ e mina d'água $(\mathrm{N}=1)$. Tais locais podem ser freqüentados por um único exemplar ou por vários animais, tendo sido capturados, simultaneamente, 11 exemplares ao saírem ou entrarem em poleiro de alimentação localizado no interior de uma manilha (cerca de $40 \mathrm{~cm}$ de diâmetro). Um dos poleiros de alimentação, situado em oco, esteve em uso por mais de 48 meses.

\section{Principais presas}

Insetos são as presas mais freqüentes entre os restos encontrados nos poleiros de alimentação. Quatro espécies de vertebrados foram até o momento identificados entre os restos (Tab. III).

Este morcego emprega os poleiros de alimentação uma ou mais vezes durante a noite, carregando na boca para estes as presas, tão logo são apreendidas. Um dos exemplares foi capturado portando uma barata ainda viva às $17: 48 \mathrm{~h}, 33$ minutos após o crepúsculo. Um exemplar foi capturado poucos minutos antes do amanhecer em redes posicionadas junto à criação de perus, pavões e galinhas, portando um besouro "rola-bosta" (Coleoptera, Scarabidae). Grande quantidade deste inseto foi observada durante a noite junto aos postes de iluminação.

Tonatia bidens forrageia tanto em bordas de matas, quanto junto a construções humanas limitrofes a bordas de matas, comprovado pela apreensão freqüente de Periplaneta americana (Linnaeus, 1758), presente em cinco dos poleiros de alimentação vistoriados.

\section{Atividade diária}

Esta espécie apresenta atividade diária compreendida entre os crepúsculos vespertino e matutino (de 33 a 638 minutos após o crepúsculo vespertino), com predominância nas primeiras horas após o pôr-do-sol. Dos 57 exemplares captura-
Tabela III. Restos de presas identificados em poleiros de alimentação onde a presença de $T$. bidens foi confirmada por captura. Os valores entre parênteses indicam o número estimado de espécies cujos restos foram encontrado

\begin{tabular}{lll}
\hline \multicolumn{1}{c}{ Ordem } & \multicolumn{1}{c}{ Família } & \multicolumn{1}{c}{ Restos } \\
\hline Lepidoptera (16) & $\begin{array}{l}\text { Arctiidae } \\
\text { Nymphalidae }\end{array}$ & Asas, tórax e espirotromba \\
Orthoptera (2) & $\begin{array}{l}\text { Mantidae } \\
\text { Scarabidae }\end{array}$ & Asas \\
Coleoptera (10) & $\begin{array}{l}\text { Cerambicidae } \\
\text { Eeros, antenas e tórax }\end{array}$ & Asas \\
Hemiptera & Belastomidae & Asas \\
Odonata & & Asas
\end{tabular}

Blattidae (4)

Anura

Squamata

Gekkonidae

Asas, tórax, patas e antenas Pélvis e membros traseiros

Passeriformes (4) Emberezidae Parte do rostrum e cauda Parte do rostrum, retrizes e membros posteriores

Chiroptera Vespertilionidae Asas, porção posterior incluindo cauda

dos com redes fora dos seus poleiros de alimentação, apenas sete (14,9\% das capturas) foram capturados após as 00:00 h, e a média do horário de capturas foi de 190,70 \pm 169,03 minutos após o pôr-do-sol (Fig. 2).

Em $22,86 \%$ das noites de coleta foram capturados mais de um exemplar simultaneamente na mesma rede, sendo observados dois $(\mathrm{N}=2)$, três $(\mathrm{N}=3)$ ou mesmo quatro $(\mathrm{N}=1)$ morcegos juntos.

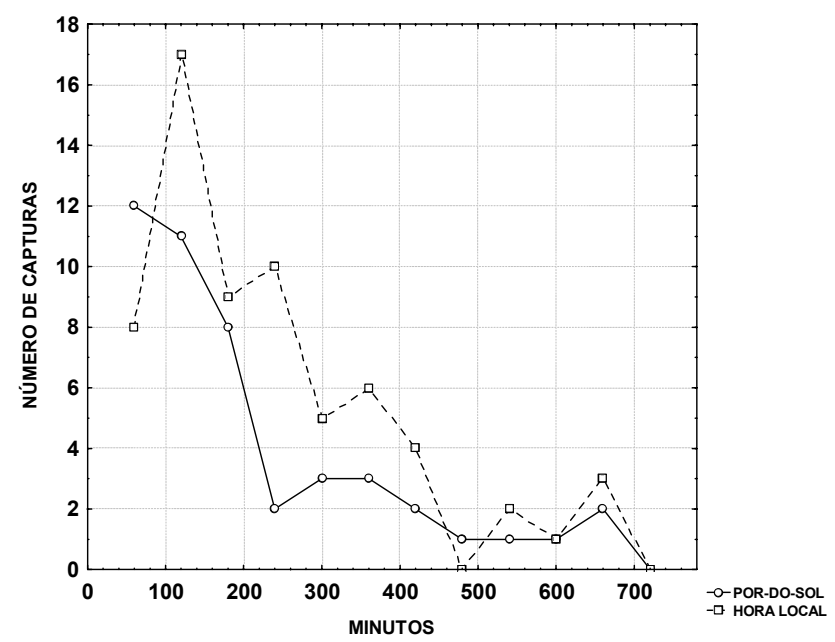

Figura 2. Horário local (a partir de 17:30 h) e minutos após o pôrdo-sol de captura de 57 exemplares de Tonatia bidens, separados a intervalos de 60 minutos. 


\section{Observações em cativeiro}

Para a captura da presa, Tonatia bidens pousa sobre esta, imobilizando-a com mordida aplicada, preferencialmente no pescoço ou cabeça. Após a apreensão o morcego voa, carregando a presa, até o teto da gaiola. Após pousar, abraça a presa com os membros anteriores, envolvendo-a com as asas. Os polegares ajudam na imobilização. A ingestão inicia-se sempre pela cabeça, sofrendo a presa uma rotação, se necessária, realizada com os dentes e os polegares.

As vísceras de pequenas presas são inteiramente ingeridas. Quando oferecidos camundongos adultos (até $25 \mathrm{~g}$ ) o morcego rejeita o terço posterior da carcaça, a cauda, o rostrum e, eventualmente, parte do intestino e estômago. Camundongos mais jovens desprovidos de pelos podem ser totalmente ingeridos. Insetos grandes como Orthoptera ou Blattariae e Lepidoptera oferecidos podem ser apreendidos pela cabeça, tórax ou abdome porém desprezam estas partes e ingerem somente o abdome. Outros insetos de médio ou grande porte quando oferecidos vivos foram prontamente ingeridos, como bicho-pau (Phasmidae), vagalume (Lampyridae) e cigarras (Cicadidae), sendo descartados pernas e asas. Exemplares pequenos de anfíbios oferecidos vivos, como Eleutherodactylus binotatus (Spix, 1824) (Leptodactilidae) e Hyla faber Wied, 1821 (Hylidae) foram aceitos, sendo desprezados parte dos membros posteriores. Um exemplar de Lagarto-de-coleira, Tropidurus torquatus (Wied-Neuwied, 1820) (Tropiduridae), de pequeno porte, $11 \mathrm{~g}$, oferecido, foi capturado, sendo ingeridos a cabeça, membros anteriores e parte das vísceras. Exemplares de lagartixas, Hemidactylus mabuia (Moreau de Jonnès, 1818) (Gekkonidae), foram ingeridos, restando parte terminal da cauda e eventualmente parte do rostro. Exemplares de bico-de-lacre, Estrilda astrid (Linnaeus, 1758) (Estrildidae), foram capturados e ingeridos, sendo desprezados membros posteriores e parte das asas, enquanto um exemplar adulto de pardal, Passer domesticus (Linnaeus, 1758) (Emberezidae), foi ignorado. Um morcego, Myotis nigricans (Schinz, 1821) (Vespertilionidae), com 3,6 g de peso, oferecido vivo, foi apreendido e teve a cabeça e parte do tórax ingerida, sendo o restante descartado. Um exemplar vivo de Molossus molossus (Pallas, 1766) (Chiroptera, Molossidae), com 9,1 g de peso não foi atacado, mesmo tendo permanecido por duas noites na gaiola. Frutos oferecidos, como banana, maça, goiaba, mamão e laranja, não foram consumidos.

\section{DISCUSSÃO}

Tonatia bidens aparenta ser comum e com ampla ocorrência no Estado do Rio de Janeiro, apesar de ser raramente citado em levantamentos de fauna do sudeste do Brasil. Tal fato pode ser explicado pela não consideração dos poleiros de alimentação como locais apropriados para captura da espécie ou pela dificuldade dos pesquisadores em localizar estes locais. Aparentemente esta espécie está restrita ou é mais freqüente em bordas de florestas secundárias e primárias. Mesmo no bioma da Caatinga as capturas de espécies de Tonatia foram restritas a vegetação mais alta (Willig 1983). Fenton et al. (1992) e Medellin et al. (2000) propuseram os morcegos como bioindicadores, baseados na associação de espécies de Phyllostominae com florestas primárias e a variação das espécies em locais com diferentes graus de perturbação ambiental. Entretanto, esta espécie utiliza-se de clareiras para forrageio, onde predominaram as capturas analisadas. A predação freqüente de espécies comensais humanas (eg. Periplaneta americana) e o uso de construções humanas limitrofes a mata para poleiro de alimentação, sugerem que pode ser influenciada positivamente com a ocupação residencial, não devendo, portanto, ser considerada isoladamente como espécie indicadora da integridade florestal.

Fêmeas ativas sexualmente foram capturadas na primavera e verão no Estado do Rio de Janeiro, ocorrendo os nascimentos, predominantemente, nos meses de novembro a janeiro e podendo ser encontradas fêmeas em final de lactação até maio. A espécie é poliestral, com reprodução estacional, sendo sugerido padrão bimodal, com o primeiro pico de nascimentos na época chuvosa (Genoways \& Williams 1984, WiLson 1979), similar a outras espécies do gênero Tonatia (veja NowaK 1995, WILSON 1979).

No Estado do Rio de Janeiro este morcego foi encontrado refugiando-se durante o dia em grutas, minas d'água e ocos de árvores. Tutle (1976), Villa-R. (1966), Eisenberg \& Redford (1999) descreveram o uso de oco de árvores para refúgio por esta espécie e outras espécies do gênero, podendo ocorrer a coabitação com outras espécies. No presente estudo foi comprovada a coabitação com outras espécies de morcegos, como T. silvicola, C. perspicillata e D. rotundus. Até sete animais da mesma espécie, dos quais seis fêmeas, foram observados utilizando o mesmo refúgio, fato que concorda com o descrito por outros autores para morcegos do gênero Tonatia, que formam pequenos grupos de até 10 animais (Tutle 1976, Fenton \& Kunz 1977). A captura de vários indivíduos simultaneamente, sugere que este morcego pode voar em grupo.

Os poleiros de alimentação são empregados por um único indivíduo ou por um grupo, variando o número capturado por noite em até 11 exemplares em cada poleiro amostrado com auxílio de redes de espera. O poleiro de alimentação pode ser empregado por grandes períodos de tempo (24 meses ou mais).

Diversos autores mencionam que a dieta do gênero Tonatia é composta por frutos e insetos capturados durante o vôo, sobre o substrato ou na vegetação (e.g. Goodwin \& Greenhall1961, GARDNER 1977). A carnivoria em Tonatia bidens foi descrita recentemente (BARquez et al. 1999, Martuscelli 1995) e aparentemente não é tão rara. As presas utilizadas por T. bidens devem ter seu peso máximo próximo ao peso do morcego, haja visto o comportamento de apreensão e manipulação nos poleiros de alimentação. Freeman (2000) comparando diferentes taxa de espécies de morcegos, afirmou que insetívoros adotam a carnivoria com o aumento da biomassa. Tonatia bidens provavelmente apresenta maior freqüência de carnivoria que as demais espécies de Phyllostominae menores (McNAB 2003) e menor que Trachops cirrhosus (Spix, 1823) e Chrotopterus auritus (Peters, 1856).

Revista Brasileira de Zoologia 21 (2): 253-259, junho 2004 
Tonatia bidens apresentou comportamento em cativeiro de apreensão e manipulação da presa similar ao já descrito para outros Phyllostomidae carnívoros - T. cirrhosus e C. auritus (McNab 2003, Peracchi \& Albuquerque 1976, Peracchi et al. 1982). A presa é apreendida no piso da gaiola e manipulada em lugar alto, imobilizada através de mordedura e pelos polegares. Tonatia bidens é espécie de hábito catador ("gleaner"), que utiliza grandes artrópodes e pequenos vertebrados em sua dieta, capturados sobre o substrato ou na vegetação (Goodwin \& Greenhall 1961, Gardner 1977, Myers \& Wetzel 1983).

\section{AGRADECIMENTOS}

É necessário o agradecimento ao Instituto Estadual de Florestal, Instituto Brasileiro de Meio Ambiente, ao Centro de Primatologia do Rio de Janeiro, as Fazendas Reunidas São João, a Reserva Ecológica Rio das Pedras, a Secretaria Municipal de Meio Ambiente do Município do Rio de Janeiro e a Secretaria Municipal de Casimiro de Abreu pela permissão para coletas e apoio concedidos. Ao Dr. Gustavo Graciolli pela identificação dos ectoparasitas coletados e ao Dr. Adriano Peracchi pela confirmação da espécie de morcego. À Dra Cristina Nassar, Dr. Nélio R. dos Reis, André Pol e Marco Aurélio Ribeiro de Mello e a dois revisores anônimos agradecemos as valiosas críticas. Parte dos recursos necessários para o desenvolvimento deste procedimento foram providos pelo Fundo de Conservação Ambiental (Processo número 14/001.917/97).

\section{REFERÊNCIAS BIBLIOGRÁFICAS}

ANTHONy, E.L.P. 1988. Age determination in bats, p. 47-58. In: T.H. Kunz (Ed.). Ecological and behavioral methods for the study of bats. Washington, DC, Smithsonian Institution Press, 533p.

Barquez, R.M.; M.A. Mares \& J.K. Braun. 1999. The bats of Argentina. Special Publications, Museum Texas Tech University, Lubbock, 42: 1-275.

EIsenberG, J.F. \& K.H. RedFord. 1999. Mammals of the neotropics. The central neotropics. Ecuador, Peru, Bolivia, Brazil. Chicago, University of Chicago Press, vol. 3, 609p.

EsBÉRARD, C. 2002. Round-eared Bat (Tonatia bidens) in captivity. Bat Research News, New York, 43 (1): 7-8.

Esbérard, C.E.L. \& C. DaEmon. 1999. Novo método para marcação de morcegos. Chiroptera Neotropical, Brasília, 5 (1-2): 116-117.

Esbérard, C. \& L.H. Gomes. 2001. Order Chiroptera (Bats), p. 219224. In: W.E. Fowler \& Z.S. CubAs (Eds). Biology, Medicine, and Surgery of South American Wild Animals. Iowa State University Press, 536p.

Fenton, M.B. \& T.H. Kunz. 1977. Movements and behavior, p. 351-364. In: R.J. Baker; J.K. Jones \& D.C. Carter (Eds). Biology of bats of the New World Family Phyllostomatidae. Part II. Special Publications Museum Texas Tech University, Lubbock, 13: 1-364.
Fenton, M.B.; L. Acharya; D. Audet; M.B.C. Hickey; C. Merriman; M.K. Овrist; D.M. Syme \& B. AdKIns. 1992. Phyllostomid bats (Chiroptera: Phyllostomidae) as indicators of habitat disruption in the Neotropics. Biotropica, Saint Louis, 24 (3): 440-446.

Freeman, P.W. 2000. Macroevolution in microchiroptera: recoupling morphology and ecology with phylogeny. Evolutionary Ecology Research, Tucson, 2: 317-355.

Gardner, A.L. 1977. Feeding Habits, p. 293-350. In: R.J. BAKER; J.K. Jones \& D.C. Carter (Eds). Biology of bats of the New World Family Phyllostomatidae. Part II. Special Publications Museum Texas Tech University, Lubbock, 13: 1-364p.

Genoways, H.H. \& S.L. Williams. 1984. Results of the Alcoa Foundation - Suriname Expeditions. IX. Bats of the genus Tonatia (Mammalia: Chiroptera) in Suriname. Annals Carnegie Museum, Pittsburgh, 53 (11): 327-346.

Goodwin, G.G. \& A. M. Greenhall. 1961. A review of the bats of Trinidad and Tobago. Bulletin of American Museum of Natural History, New York, 2 (3): 187-302.

Martuscelli, P. 1995. Avian predation by Round-eared bat (Tonatia bidens, Phyllostomidae) in the Brazilian Atlantic Forest. Journal Tropical Ecology, Cambridge, 11: 461-464.

$\mathrm{MCN}_{\mathrm{AB}}$, B.K. 2003. Standart energetics of phyllostomid bats: the inadequacies of phylogenetic-contrast analyses. Comparative Biochemistry and Physiology Part A 135: 357-368.

Medellín, R.A.; M. Equinua \& M.A. Almin. 2000. Bat diversity and abundance as indicators in Neotropical Forests. Conservation Biology, Alameda, 14 (6): 1666-1675.

Myers, P \& R.M. Wetzel. 1983. Systematics and zoogeography of the bats of Chaco Boreal. Miscellaneous Publications of The Museum of Zoology, Michigan, 165: 1-59.

NOWAK, R.M. 1995. Walker's mammals of the world. Baltimore, John Hopkins University Press, $4^{\text {th }}$ ed., 1629 p.

Peracchi, A.L. \& S.T. Albuquerque. 1976. Sobre os hábitos alimentares de Chrotopterus auritus australis Thomas, 1905 (Mammalia: Chiroptera: Phyllostomidae). Revista Brasileira de Biologia, Rio de Janeiro, 36 (1): 179-184.

ـ 1986. Quirópteros do Estado do Rio de Janeiro, Brasil (Mammalia, Chiroptera). Publicações Avulsas do Museu Nacional, Rio de Janeiro, 66: 63-69.

Peracchi, A.L.; S.T. Albuquerque \& S.D.L. Raimundo. 1982. Contribuição ao conhecimento dos hábitos alimentares de Trachops cirrhosus (Spix, 1823) (Mammalia: Chiroptera: Phyllostomidae). Arquivos da Universidade Federal Rural do Rio de Janeiro, Rio de Janeiro, 5 (1):1-5.

PINTo-DA-Rocha, R. 1995. Sinopse da fauna cavernícola do Brasil (1907-1994). Papéis Avulsos de Zoologia, São Paulo, 39 (6): 61-173.

Ruschi, A. 1953. Morcegos do Estado do Espírito Santo. XIX. Família Phyllostomidae, descrição de Tonatia bidens e Sturnira lilium. Família Vespertilionidae, descrição de Eptesicus hilarii e Eptesicus brasiliensis. Família Molossidae, descrição de 
Eumops abrasus abrasus. Com algumas observações a respeito. Boletim Museu Biologia Professor Mello Leitão, Santa Teresa, 21: 1-8.

TutLe, M.D. 1976. Collecting techniques, p. 71-88. In: R.J. BAKER; J.K. Jones \& D.C. Carter (Eds). Biology of bats of the new world family Phyllostomidae. Part 1 . Special Publications Museum Texas Tech University, Lubbock, 10: 1-324.

VieIRA, C.O.C. 1942. Ensaio monográfico sobre os quirópteros do Brasil. Arquivos Zoologia de São Paulo, São Paulo, 3: 371-387.

VILLA-R, B. 1966. Los murciélagos de México. México, Instituto de Biologia, Universidad Nacional de México, 492p.

WhitAKER JR., J.O. 1988. Collecting and preserving ecotoparasites for ecological studies, p. 459-474. In: T.H. KunZ (Ed.). Ecolo- gical and behavioral methods for the study of bats. Washington, DC, Smithsonian Institution Press, 533p.

Williams, S.L.; M.R.Willig \& F.A. Reid. 1995. Review of the Tonatia bidens complex (Mammalia: Chiroptera), with description of two new subspecies. Journal of Mammalogy, Baltimore, 76 (2): 616-726.

WILLIG, M.R. 1983. Composition, microgeographic variation, and sexual dimorphism in Caatingas and Cerrado biomes in northeast Brazil. Journal of Mammalogy, Baltimore, 66 (4): 668-681.

WILSON, D. 1979. Reproductive patterns, p. 317-378. In: R.J. BAKER; J.K. Jones \& D.C. Carter (Eds). Biology of bats of the new world family Phyllostomidae. Part 1. Special Publications, Museum Texas Tech University, Lubbock, 10: 1-324p.

Recebido em 29.VIII.2003; aceito em 16.V.2004. 